\title{
Interrelación entre nivel de autoestima de adolescentes y nivel de disfunción familiar. Distrito de Hunter, Arequipa, 2007
}

\section{Interrelation of Adolescent Self-Esteem Level and Level of Family Dysfunction. Hunter Dis- trict, Arequipa, 2007}

\author{
Cristhian José Vargas Lazo* \\ http://dx.doi.org/10.21503/CienciayDesarrollo.2010.v12.06
}

\section{RESUMEN}

La familia tiene estructuras y funciones diversas que difieren en los distintos países y culturas. Esta rica variedad de las estructuras familiares es consecuencia de opciones individuales y de valores sociales. Las estructuras y funciones familiares cambian y se adaptan constantemente a las tendencias sociales y del ambiente exterior. Sin embargo, cualesquiera que sean los cambios, el concepto de familia sobrevive como unidad social importante en casi todas las sociedades.

Autoestima es la capacidad desarrollable de experimentar la existencia, conscientes de nuestro potencial y nuestras necesidades reales, de amarnos incondicionalmente y confiar en nosotros para lograr objetivos, independientemente de las limitaciones que podamos tener o de las circunstancias externas generadas por los distintos contextos en los que nos corresponda interactuar.

La autoestima es una de las bases para el éxito personal y factor protector para diversas patologías de la salud mental. Puesto que la adolescencia es una etapa de cambios significativos tanto físicos como psicológicos, puede pensarse que se trata de un período de alta vulnerabilidad, donde se es más propenso a presentar alteraciones en la autoestima y a desarrollar patologías de salud mental (adicciones, trastornos de la conducta alimentaria, depresión, etc.).

En el presente trabajo encontramos que se puede considerar a la disfunción familiar como un factor interventor en el desarrollo de la autoestima, y como un aspecto negativo (factor de riesgo) para la autoestima de los adolescentes. Se halló también que los adolescentes con niveles de baja autoestima presentan grados de disfunción familiar, lo que debería ser estudiado más profundamente.

Palabras clave: familia, adolescencia, autoestima, disfunción familiar.

\section{ABSTRACT}

The family has structures and diverse functions that differ in the different countries and cultures. This rich variety of the family structures is consequence of individual options and of social values. The structures and family functions change and they constantly adapt to the social tendencies and of the external atmosphere. However, any that you/they are the changes, the family concept survives as important social unit in almost all the societies.

Self-esteem is the capacity we can develop to experience the existence, aware of our potential and our real necessities; of to love us unconditionally and to trust us to achieve objectives, independently of the limitations that we can have or of the external circumstances generated by the different contexts in those

*Docente de la Escuela Académico-Profesional de Farmacia y Bioquímica de la Universidad Alas Peruanas, filial Arequipa. 
that it corresponds us to interact.

Being the Self-esteem one of the bases for the personal success and protective factor for diverse pathologies of the mental health; Since the adolescence is a stage of significant changes so much physical as psychological, it can be thought that is of a population of high vulnerability, being this way prone to present alterations in its Self-esteem and risk of development of pathologies of mental health (addictions, dysfunctions of the behavior would feed, depression, etc.)

Presently work finds that you can consider the family Dysfunction as a factor inspector in the development of the Self-esteem, in the negative aspect (risk factor) for the Self-esteem of the Adolescents. That the adolescents that present levels of bad Self-esteem have in relationship direct degrees of family dysfunction. That should be studied more deeply.

Key Words: family, adolescence, self-esteem, family dysfunction.

\section{INTRODUCCIÓN}

La adolescencia es la etapa de la vida comprendida entre los 12 y 18 años ${ }^{16}$. Diversos investigadores han contribuido a la definición más integral de la adolescencia. Según Piaget, es la "etapa de operaciones formales" del desarrollo cognoscitivo. Erickson identifica dos tareas diferentes para la adolescencia: la primera de ellas es "identidad versus difusión de rol”, y la segunda, "intimidad versuss aislamiento", como parte del desarrollo personal-social. Para Freud, es la "etapa genital" de las etapas psicosexuales del desarrollo.

El presente trabajo de investigación nace por la inquietud de la práctica cotidiana y el trabajo realizado con adolescentes, en donde encontramos que la gran mayoría de adolescentes con baja autoestima provenían de hogares disfuncionales.

Por ello se revisan antecedentes investigativos, hallándose muy pocos a nivel internacional, y ninguno a nivel local, que remarquen específicamente este punto la interrelación entre los niveles de autoestima y el nivel de funcionabilidad familiar.

La autoestima es una de las bases para el éxito personal y factor protector para diversas patologías de la salud mental. Puesto que la adolescen- cia es una etapa de cambios significativos tanto físicos como psicológicos, puede pensarse que se trata de un período de alta vulnerabilidad, donde se es más propenso a presentar alteraciones en la autoestima y a desarrollar patologías de salud mental (adicciones, trastornos de la conducta alimentaria, depresión, etc.). Creo importante, por ello, la realización de esta investigación.

\section{MATERIAL Y MÉTODO}

El presente estudio se realizó en el mes de noviembre del 2007, en el distrito de Hunter. Se trabajó con 50 adolescentes que se constituyeron en la muestra por conveniencia, elegidos al azar (entre voluntarios que se presentaron ante solicitud en la marquesina del centro del distrito), cumpliendo los criterios de inclusión y exclusión.

\section{Instrumentos}

- Ficha de recolección de datos, la cual contiene:

Test de autoestima de Coersmith

Test de Apgar familiar

\section{Criterios de inclusión}

- Tener entre 12 y 18 años de edad.

- Estar dispuesto a colaborar con el estudio.

- Contar con el consentimiento informado de los padres para el estudio. 


\section{Criterio de exclusión}

- Padecer de algún trastorno mental.

\section{Tamaño y diseño de la muestra}

La población son los adolescentes del distrito de Hunter de la ciudad de Arequipa, y solo se trabajará con 50 de ellos, que se constituirán en la muestra por conveniencia, elegidos al azar (voluntarios presentados ante solicitud en la marquesina del centro del distrito), cumpliendo los criterios de inclusión y exclusión.

\section{Validación de los instrumentos}

El Test de Cooersmith ha sido validado por los diferentes estudios que aceptan su uso co- tidiano en el estudio de la autoestima.

El Test de Apgar Familiar ha sido validad por los diferentes estudios que lo aceptan como parte de la evaluación de la funcionalidad familiar.

\section{Criterios o estrategias para el manejo de los} resultados

Una vez que se obtuvieron los datos, estos fueron procesados, tabulados y analizados haciendo un análisis inductivo-deductivo, utilizando estadística descriptiva y las pruebas estadísticas Chi cuadrado, P (significancia estadística) y Odds Ratio, permitiendo lograr las conclusiones de la presente investigación.

\section{RESULTADOS}

Tabla 1. Muestra según género y edad

\begin{tabular}{|c|c|c|c|c|c|c|c|c|c|c|c|c|}
\hline \multicolumn{13}{|c|}{ Muestra adolescentes - Hunter } \\
\hline Edad & \multicolumn{2}{|c|}{12} & \multicolumn{2}{|c|}{13} & \multicolumn{2}{|c|}{14} & \multicolumn{2}{|c|}{15} & \multicolumn{2}{|c|}{16} & \multicolumn{2}{|c|}{ TOTAL } \\
\hline Genero & $\mathrm{N}^{\circ}$ & $\%$ & $\mathrm{~N}^{\circ}$ & $\%$ & No & $\%$ & $\mathrm{~N}^{\circ}$ & $\%$ & $\mathrm{~N}^{\circ}$ & $\%$ & $\mathrm{~N}^{\circ}$ & $\%$ \\
\hline Hombre & 3 & 6,00 & 5 & 10,00 & 6 & 12,00 & 7 & 14,00 & 7 & 14,00 & 28 & 56,00 \\
\hline Mujer & 2 & 4,00 & 4 & 8,00 & 4 & 8,00 & 5 & 10,00 & 7 & 14,00 & 22 & 44,00 \\
\hline TOTAL & 5 & 10,00 & 9 & 18,00 & 10 & 20,00 & 12 & 24,00 & 14 & 28,00 & 50 & 100,00 \\
\hline
\end{tabular}

En el presente cuadro encontramos que el $56 \%$ de nuestra muestra es de género masculino. Además, el mayor porcentaje se sitúa en la edad de16 años.

Gráfico 1. Muestra según género y edad

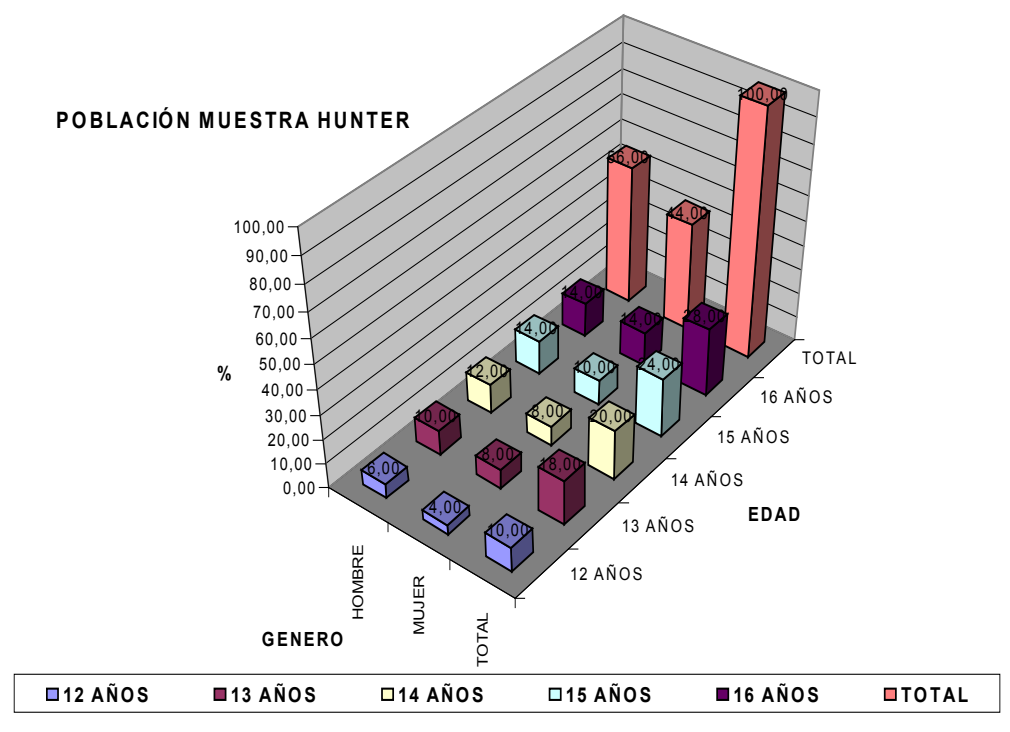


Tabla 2. Autoestima

\begin{tabular}{|c|c|c|c|c|c|c|c|}
\hline \multicolumn{8}{|c|}{ MUESTRA HUNTER } \\
\hline & \multirow{2}{*}{ Género } & \multicolumn{2}{|c|}{ Hombre } & \multicolumn{2}{|c|}{ Mujer } & \multicolumn{2}{|c|}{ TOTAL } \\
\hline Autoestima & & $\mathrm{N}^{\circ}$ & $\%$ & $\mathrm{~N}^{\circ}$ & $\%$ & $\mathrm{~N}^{\circ}$ & $\%$ \\
\hline Muy mala & de 0 a 6 & 3 & 10,71 & 2 & 9,09 & 5 & 10,00 \\
\hline Mala & de 7 a 13 & 13 & 46,43 & 10 & 45,45 & 23 & 46,00 \\
\hline Buena & de 14 a 19 & 3 & 10,71 & 5 & 22,73 & 8 & 16,00 \\
\hline Muy buena & de 20 a 25 & 9 & 32,14 & 5 & 22,73 & 14 & 28,00 \\
\hline \multicolumn{2}{|c|}{ TOTAL } & 28 & 100,00 & 22 & 100,00 & 50 & 100,00 \\
\hline
\end{tabular}

Mínima autoestima: 0

Máxima autoestima: 25

En la tabla anterior apreciamos que el mayor porcentaje de la muestra presenta niveles de mala autoestima (46\%), seguido de muy buena autoestima (28\%).

Grafico 2. Autoestima

AUTOESTIMA

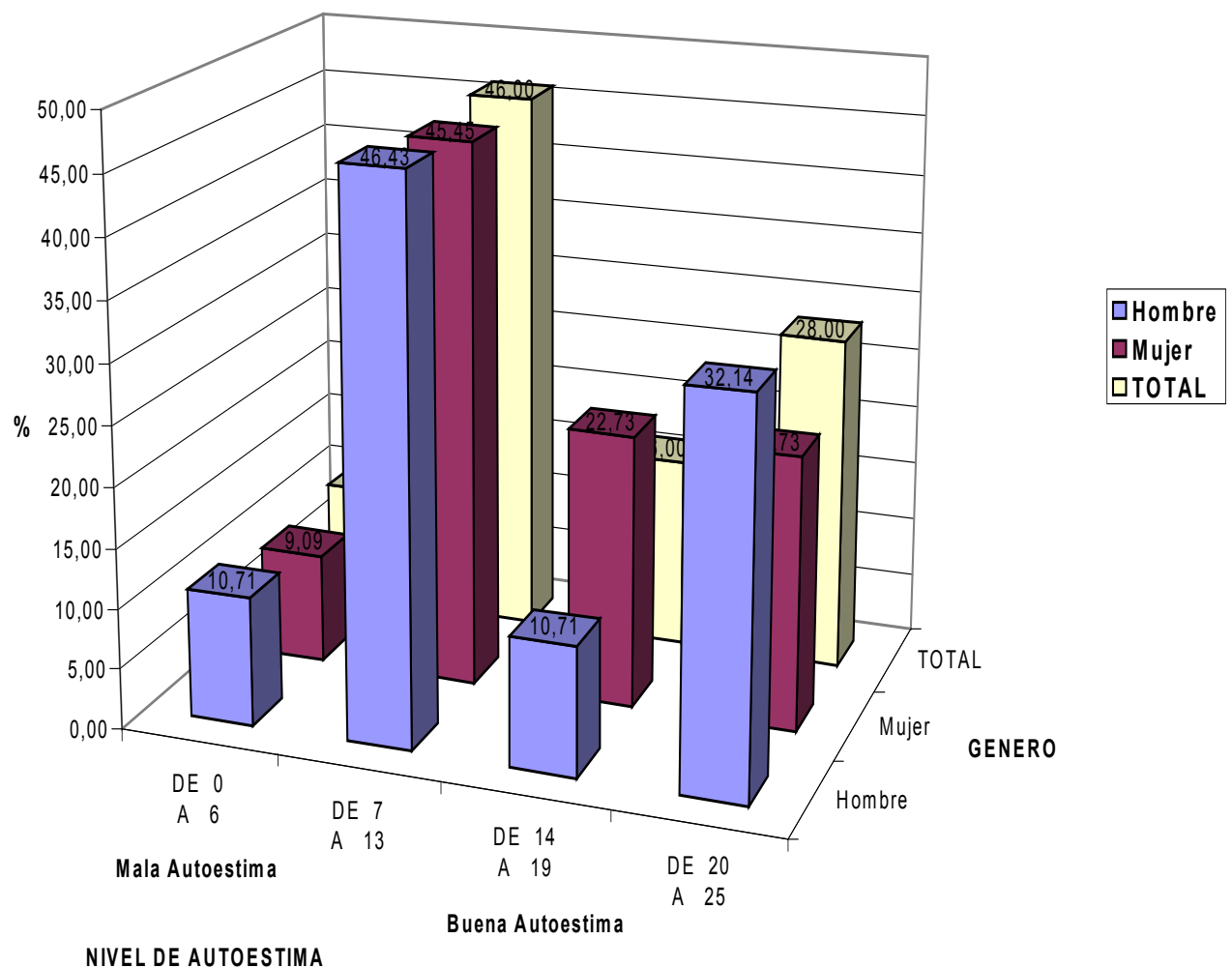


Tabla 3. Disfunción familiar

\begin{tabular}{|l|c|c|c|c|c|r|}
\hline \multirow{2}{*}{ Género } & \multicolumn{2}{c|}{ Hombre } & \multicolumn{2}{c|}{ Mujer } & \multicolumn{2}{c|}{ TOTAL } \\
\cline { 2 - 8 } & $N^{\circ}$ & $\%$ & $N^{\circ}$ & $\%$ & $N^{\circ}$ & $\%$ \\
\hline $\begin{array}{l}\text { Disfunción } \\
\text { familiar }\left(^{*}\right)\end{array}$ & 13 & 46,43 & 10 & 45,45 & 23 & 46,00 \\
\hline $\begin{array}{l}\text { Sin } \\
\text { disfunción }\end{array}$ & 9 & 32,14 & 6 & 27,27 & 15 & 30,00 \\
\hline $\begin{array}{l}\text { Disfunción familiar } \\
\text { Disfunción severa }\end{array}$ & 6 & 21,43 & 6 & 27,27 & 12 & 24,00 \\
\hline \multicolumn{1}{c|}{ TOTAL } & 28 & 100,00 & 22 & 100,00 & 50 & 100,00 \\
\hline
\end{tabular}

0 a 3 = disfunción familiar severa

3 a $6=$ disfunción familiar

$>6=$ funcionamiento familiar adecuado

En la tabla anterior apreciamos que el $54 \%$ de la muestra presenta disfunción familiar, menor porcentaje en el nivel severo e igual en proporción en hombres que en mujeres. El 23\% de la muestra presenta un funcionamiento familiar adecuado.

\section{Grafico 3. Disfunción familiar}

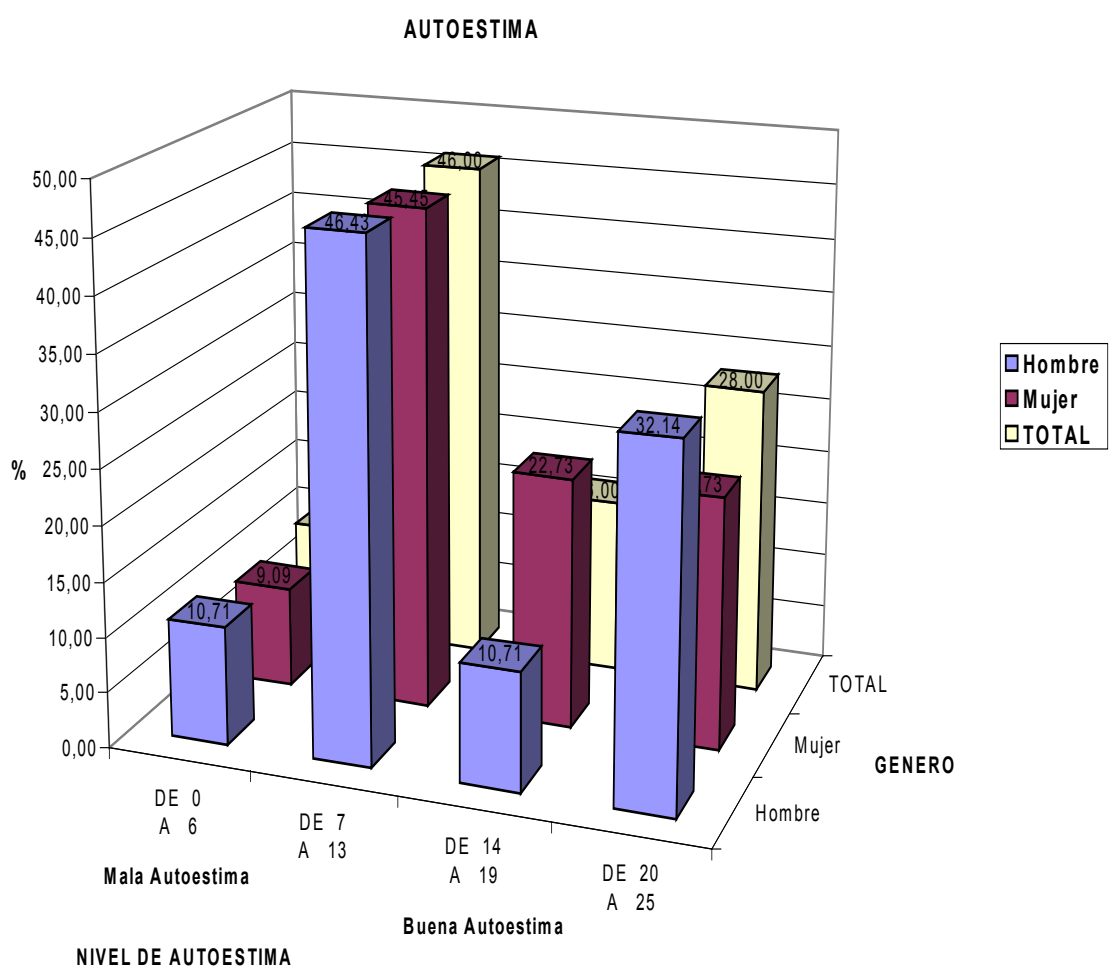


Tabla 4. Autoestima versus disfunción familiar

\begin{tabular}{|c|c|c|c|c|c|c|}
\hline \multicolumn{7}{|c|}{ MUESTRA HUNTER } \\
\hline Autoestima & \multicolumn{2}{|c|}{ Hombre } & \multicolumn{2}{|c|}{ Mujer } & \multicolumn{2}{|c|}{ TOTAL } \\
\hline Disfunción & $\mathrm{N}^{\circ}$ & $\%$ & $\mathrm{~N}^{\circ}$ & $\%$ & $\mathrm{~N}^{\mathrm{o}}$ & $\%$ \\
\hline $\begin{array}{l}\text { Sin } \\
\text { disfunción }\end{array}$ & 2 & 7,14 & 21 & 95,45 & 23 & 46,00 \\
\hline Disfunción familiar & 26 & 92,86 & 1 & 4,55 & 27 & 54,00 \\
\hline TOTAL & 28 & 100,00 & 22 & 100,00 & 50 & 100,00 \\
\hline
\end{tabular}

Chi cuadrado

7,9368

$\mathrm{P}$ (significancia estadística) $\quad 0,0473$

Odds Ratio

En la tabla de arriba apreciamos la interrelación entre la autoestima y la disfunción familiar. Se observa un mayor porcentaje de población con mala autoestima y disfunción familiar. El Odds Ratio de 0,0037 nos muestra una relación de factor de riesgo de la disfunción familiar sobre la autoestima.

Gráfico 4. Autoestima versus disfunción familiar

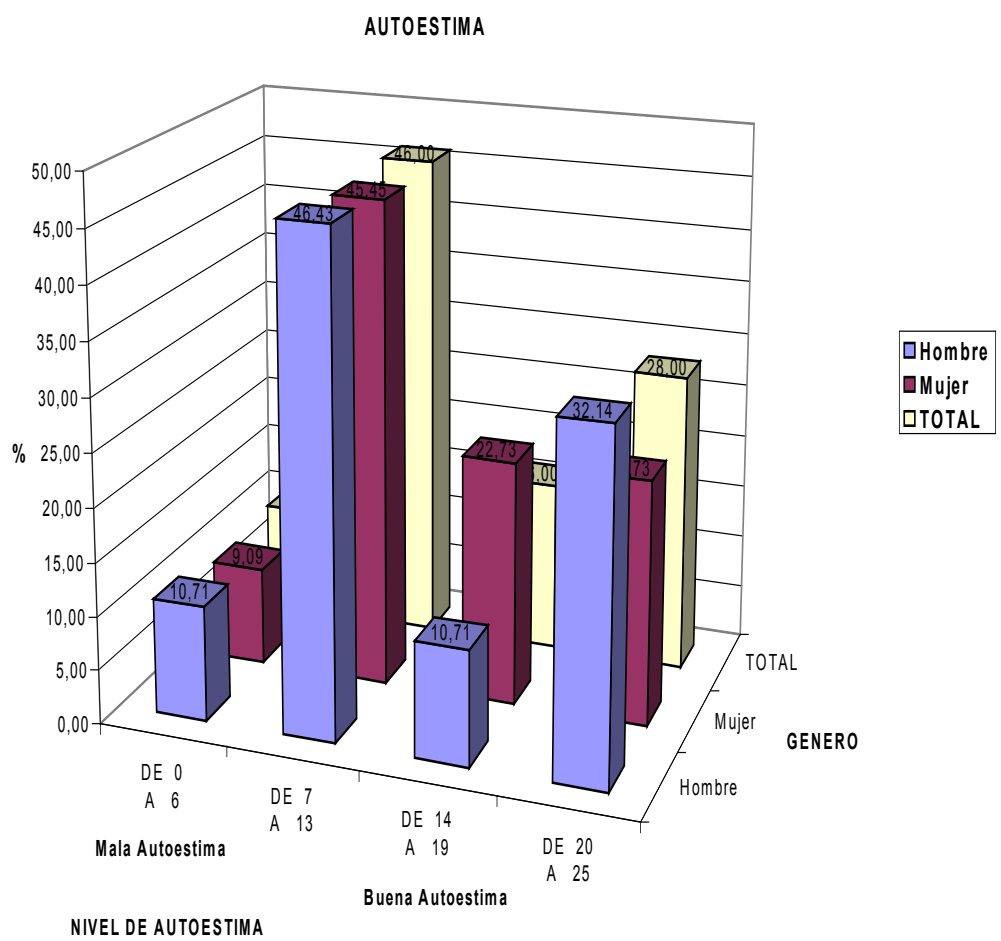




\section{DISCUSIÓN}

Como decíamos, no hay evidencias bibliográficas de trabajos similares. En nuestro trabajo hallamos una diferencia entre los hallazgos entre géneros, ya que los hombres presentan mejores niveles de autoestima y las mujeres provienen en mayor proporción de hogares disfuncionales. Todo ello lleva a apoyar nuestros resultados de que la disfunción familiar influye decisivamente en el desarrollo de la autoestima en niños y en su consolidación durante la adolescencia.

Se debería trabajar con poblaciones más extensas para generalizar los hallazgos y realizar programas de intervención.

\section{CONCLUSIONES}

- Primera. El mayor porcentaje de la muestra presenta niveles de mala autoestima (46\%), seguido de muy buena autoestima (28\%)

- Segunda. El 54\% de la muestra presenta disfunción familiar, siendo ello este porcentaje alto. El menor porcentaje se da en el nivel severo, e igual en proporción en hombres que en mujeres. El 23\% de la muestra presenta un funcionamiento familiar adecuado.

- Tercera. Hay mayor porcentaje de población con mala autoestima y disfunción familiar.

- Cuarta. Sí existe relación entre la disfunción familiar y la autoestima, y esa relacion es negativa, pudiéndose considerar como un factor de riesgo. El Odds Ratio es de 0,0037.

\section{RECOMENDACIONES}

- Primera. Tener una evaluación periódica de los niveles de aptitud social, como autoestima, asertividad y resiliencia en nuestros adolescentes.

- Segunda. Considerar la disfunción familiar como factor de riesgo de la autoestima en adolescentes.
- Tercera. Ampliar los estudios a poblaciones similares para obtener una muestra más significativa.

- Cuarta. Prestar atención a la promoción del desarrollo de la autoestima desde la infancia.

- Quinta. Proponer programas de intervención familiar para mejorar las interrelaciones personales y la calidad de vida del producto de la familia: los hijos.

\section{AGRADECIMIENTOS}

Al personal de salud de la Municipalidad de Hunter por su apoyo al presente trabajo, así como a los adolescentes que participaron de manera voluntaria y veraz en el presente trabajo.

A mi familia por su apoyo incondicional.

\section{REFERENCIAS BIBLIOGRÁFICAS}

1. Llaza Loayza, Gloria, y colaboradores. $M a-$ nual de Psiquiatría 2003. I: 53-70; XII: 145154

2. López, Juan J. y colaboradores. DSM IV TR, Manual diagnóstico y estadístico de los trastornos mentales. Masson S.A., Barcelona (España), 2002

3. Birmaher B., Ryan N.D., Williamson D.E., Brent D.A., Kaufman J., Dahl R.F., et al. Childhood and Adolescent Depression: a Review of the Past 10 Years. J Am Acad Child Adolesc Psychiatry 1996; 35:1427-39.

4. Colimon K.M. Fundamentos de epidemiología. Madrid, Díaz de Santos, 1990.

5. Belloch A., Sandin B., Ramos F. Manual de psicopatología. Madri, Interamericana - McGraw Hill, 1995.

6. Mullen D.J., Hendren R.L. "El niño o adolescente suicida”. En: Psiquiatría del niño y del adolescente. Chicago, Hartcourbrace, 1998.

7. Freeman A., Reinecke M.A. Terapia cognitiva aplicada a la conducta suicida. Bilbao, Disclée de Brouwer,m 1995. 
8. Rodríguez Estrada; Mauro, Georgina Pellicer de Flores; Magdalena Domínguez Eyssautier. Cómo desarrollar la autoestima.

9. Aberastury, A. y Knobel, M. La adolescencia normal. Editorial Paidós, Bs.As., 1976.

10. Mckay, Fanning. Autoestima: evaluación y mejora. Editorial Martínez Roca.

11. Prosperi Paola. Autostima nel contesto familiare e la influenza della disfunzione familiare. Casa editrice Cuore e Psiche, 2006

12. González Y., et al. Prevalencia de depresión en estudiantes de medicina de la Universidad de Antioquia. Medellín, 1992.

13. Polaino-Lorente A., Mediano M.L., Martínez R. Estudio epidemiológico de la sintomatología depresiva infantil en la población escolar madrileña de ciclo medio. An Esp Pediatr 1997;46:344-50.

14. Silva, Giselle. Resiliencia y violencia politica en niños. Fundación Bernard Van Leer, Universidad Nacional de Lanús, año 1999.
15. Morchio de Uano Lucio. "La construcción de autoimágenes positivas en los alumnos". Revista Novedades Educativas, No 116, año 2000.

16. Vargas Lazo Cristhian. Autoestima, asertividad y resiliencia como factores protectores de depresión en adolescentes de dos colegios de Arequipa. UCSM, 2006.

17. www.umm.edu/medref/

18. www.kerman.org

19. www.copel.com.an

20. www.cedro.org.pe

21. www.herreros.com.ar

22. www.nlm.nih.gov/medlinplus/

23. www.psicopedagoika.it

24. www.infocuore.info/htm

25. www2.ui.ah.fi/projcts/metodi/s00.htm 